Questions de communication

$24 \mid 2013$

Renouvellement des mises en scène télévisuelles de la politique

Françoise MÉNAND DOUMAZANE, Miroirs d'Aline. Ethnocritique d'un roman de C. F. Ramuz

Nancy, PUN-Éd. universitaires de Lorraine, coll. EthnocritiqueS, 2013, 349 pages

\title{
Ammar Benkhodja
}

\section{OpenEdition}

\section{Journals}

Édition électronique

URL : http://journals.openedition.org/questionsdecommunication/8766

DOI : 10.4000/questionsdecommunication.8766

ISSN : 2259-8901

\section{Éditeur}

Presses universitaires de Lorraine

Édition imprimée

Date de publication : 31 décembre 2013

Pagination : 257-258

ISBN : 978-2-8143-0182-5

ISSN : 1633-5961

Référence électronique

Ammar Benkhodja, «Françoise ménAnd doumazane, Miroirs d'Aline. Ethnocritique d'un roman de C. F.

Ramuz », Questions de communication [En ligne], 24 | 2013, mis en ligne le 01 février 2014, consulté le

10 décembre 2020. URL : http://journals.openedition.org/questionsdecommunication/8766 ; DOI :

https://doi.org/10.4000/questionsdecommunication.8766 
processus de « légitimation par les politiques culturelles des pratiques infraculturelles (rock, bande dessinée) mais aussi des goûts d'avant-garde » (p. 5I). L'extension considérable du nombre des genres culturels et du volume des œuvres est allée de pair avec une meilleure accessibilité des publics aux biens culturels de masse par les moyens techniques. En conséquence, les formes de la consommation culturelle « ont [...] évolué dans le sens de pratiques moins conventionnelles, plus ludiques, festives, participatives » (p.55). La transmission de la culture passe par un double mouvement de dissociation : d'une part, des scènes institutionnelles comme l'école ou le marché du travail et, d'autre part, des scènes sociales ordinaires de la sociabilité, celles des pairs. On explique ce mouvement de dissociation par une dialectique entre une culture de l'affichage et de la scène publique et entre une scène intime des goûts et des écoutes réelles plus éclectiques.

Dans la quatrième partie (« Faiblesses légitimistes », pp. 81-94), l'auteur pose la question de la légitimité des cultures contemporaines et prend l'exemple du rock : « l'assignation du rock à la catégorie des genres à moyenne légitimité culturelle [méconnaît] que le rock est dorénavant légitime parce que écouté par des catégories diplômées [... . et institutionnalisé aux yeux de certains » (p. 83). En conclusion, Hervé Glevarec (p. 95) souligne « l'hétérogénéité qu'il y a entre la culture et le savoir, entre le fait d'être cultivé et le fait de produire du savoir $\gg$. Cette analyse, éclairante à plus d'un titre pour le chercheur en sciences humaines et sociales, ouvre des perspectives nouvelles.

\section{Alexandre Eyries \\ 13M, université Nice Sophia Antipolis, F-06200 alex.eyries@yahoo.fr}

\section{Françoise Ménand Doumazane, Miroirs d'Aline. Ethnocritique d'un roman de C.F. Ramuz.}

Nancy, PUN-Éd. universitaires de Lorraine, coll. Ethnocritiques, 2013,349 p.

On s'est longtemps habitué à lire Aline, premier roman de Charles-Ferdinand Ramuz (Lausanne, Payot, 1905) comme la tragédie d'un personnage victime d'un innocent amour passionnel. Mais, à la lecture pluridisciplinaire, au carrefour des sciences des textes littéraires et de l'ethnologie du symbolique qu'en propose Françoise Ménand Doumazane, on apprendra vite que l'enjeu esthétique et poétique dépasse le cadre réaliste d'un drame amoureux. En effet, la chercheuse en révèle l'agora et l'architecture des voix culturelles qui s'y jouent et s'y orchestrent.
Cette professeure agrégée de lettres classiques et docteure en langue et littérature françaises explore donc l'esthétique et la poétique de Charles-Ferdinand Ramuz. Rencontrant l'esthétique picturale de Lorenzo Lotto dès la première scène du roman d'Aline, cette poétique se révèle petit à petit au long de ce parcours analytique qu'on a tant plaisir à lire grâce à un jeu de miroirs qui renvoie aux expériences sociales et scripturales de l'auteur tout en les faisant s'entrecroiser. Miroirs d'Aline s'ouvre sur une première partie intitulée «Aline en miroir » (pp. 2 | - | |4). Dans ses huit chapitres, la critique tente un parcourt « ethnobiographique », explorant les « commencements 》 de l'auteur. Ses 27 premières années sont parcourues : sa naissance et son enfance, son parcours à l'école préparatoire et ses années de collège jusqu'au moment où il commence l'expérience scripturale d'Aline. De cet ensemble d'« expériences », la chercheuse sélectionne des biographèmes dont elle propose une lecture cultuelle qui fait ressortir trois mots clés : 《 circulations 》, « passages 》 et « transactions 》. Ainsi l'ethnocritique éloigne-t-elle son approche des lectures biographiques classiques tout en mettant « en miroir

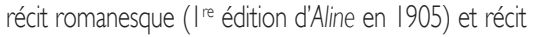

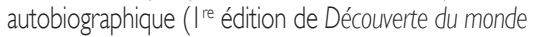
en 1993) » (p. 22). Conformément à la démarche ethnocritique, elle permet aussi de conjoindre littérature et sciences humaines. Françoise Ménand Doumazane relève aussi tous les croisements qui existent entre le texte de Charles-Ferdinand Ramuz et d'autres auteurs, tels François-René de Chateaubriand, John Berger...

La deuxième partie, « Aline au miroir 》 (pp. I 19-225), fait structurellement écho à celle qui la précède : elle est pareillement composée de huit chapitres. Ici, le miroir est orienté vers le matériau textuel qui se révèle être riche en éléments culturels. Données culturelles et symboliques que « Ramuz, par un travail d'écriture en spirale, s'approprie, réélabore et se réapproprie »(p. |19). Ainsi, s'agissant avant tout d'une étude tournée vers la littérarité du texte, l'auteure commence-t-elle par en étudier les grandes catégories narratives : espaces-temps, parcours actantiels des personnages et structure de l'intrigue y sont judicieusement décortiqués et, par la prouesse méthodologique que permet l'ethnocritique de la littérature, la chercheuse met au jour la polyphonie culturelle en œuvre. Catégorisant et nommant la configuration spatiale du roman grâce à des concepts empruntés à l'anthropologie, Françoise Ménand Doumazane a mis au point une structure ternaire lui permettant d'approcher anthropologiquement la réélaboration fictive de l'espace : la domus (espace 
familier et domestique), le seltus (terres incultes et abandonnées à la végétations) et le campus (terres maîtrisées et cultivées par l'homme) s'imbriquent avec une structure temporelle cyclique condamnant le personnage d'Aline à un destin manqué et inachevé à bien des égards. Aussi, tout au long du parcours de son parcours, une figure semble être révélatrice du destin comme de la poétique du personnage et de la culture à l'œuvre : celle de la taupe et du taupier. Le croisement du fil métaphorique qui animalise Aline et le fil symbolique de la figure de la taupe fait entendre Aline à la critique comme une passante au destin manqué, un personnage liminaire, bloqué au seuil d'un destin sans lasse manqué.

La troisième partie, « Aline, livre-miroir 》 (pp. 229326), est un retour autoreflexif tourné en une « auto-ethnologie » de l'analyste. Elle est composée de l'ensemble des notes de lectures qui figure le déroulement de son itinéraire analytique, et cela de mi-janvier 2004 à septembre 2010. Ce chapitre rend non seulement compte de l'expérience de Françoise Ménand Doumazane en tant que chercheuse, mais aussi et surtout de son expérience en tant que lectrice traversant les « champs contemporains du savoir et de la société » (p. 323), et avançant à contrecourant d'une « auto-subjectivation ».

La prouesse de la lecture érudite de Françoise Ménand Doumazane réside non seulement dans son attention minutieuse au détail de l'œuvre qui fait « surgir toute l'œuvre » (p. 322), révélant ainsi la richesse des univers orchestrés par l'écriture de Chrales-Ferdinand Ramuz, mais aussi et surtout dans la finesse d'une herméneutique qui révèle l'insolite là où l'on ne voit que du commun, du banal.

Ammar Benkhodja CREM, université de Lorraine, F-57000 a.benkhodja@gmail.com

\section{Stéphane OLIvESI, L'expérience esthétique. Une archéologie des arts et de la communication.}

Paris, H. Champion, 2012,45I p.

Le terme « archéologie » utilisé dans le sous-titre renvoie à une démarche d'historien. C'est pourquoi l'histoire de l'art est presque née en même temps que l'art lui-même. Elle s'est généralisée grâce à l'esthétique de Friedrich Hegel et le XIX siècle a commencé à systématiser à la fois la critique et les approches historiques liées à un besoin de classement et à une volonté de mettre de l'ordre là où il ne semble pas y en avoir. Ce terme d'archéologie renvoie à Michel Foucault, dont l'auteur a analysé la méthode dans un article « User et mésuser... » (accès : http:// w3.u-grenoble3.fr/les_enjeux/2004/Olivesi/olivesi.pdf, consulté le 30/08/13). Dans cet article, on retrouve déjà la même méthode utilisée dans l'ouvrage. En choisissant une démarche scientifique qu'il considère comme positiviste, l'auteur explore toutes les pistes de manière objective et rationnelle. Ce qui le conduit aussi à imaginer les limites de ce qu'il avance. II offre à penser et, ensuite, invite à critiquer en proposant une forme de dialectique habilement structurée. Cette progression heuristique le conduit d'un postulat de départ à une thèse finale qui est loin d'être une opinion figée. L'image de l'archéologie renvoie à une esthétique organique, une stylistique imagée qui repose sur une vue originale de l'art et de sa critique. Elle est donc intéressante à commenter. Elle renvoie aussi à une sorte d'architecture qui relèverait d'une vue de l'esprit à même d'assembler divers éléments composites afin de structurer un édifice cohérent. Les constituants de cet édifice sont l'histoire, le langage, l'approche de l'auteur, le génie, les sens, l'émotion, l'expérience, voire les expériences et sa dynamique.

Dans l'introduction (pp. 7-15), l'auteur du livre présente la sociologie contemporaine. Elle est un outil qui permet d'élargir la compréhension des universaux impliqués dans l'expérience artistique. L'auteur tente de définir avec une méthode imparable et une logique forte. Le livre aborde le thème selon différents points de vue. En effet, l'auteur étudie plusieurs perspectives. Il se place tantôt en philosophe, renonçant à certaines théories de la phénoménologie, tantôt en sociologue ou en historien. Ainsi précise-t-il son projet de départ. Pour lui, l'expérience esthétique est une énigme. Plusieurs rencontres en sont à l'origine. II s'agit de la rencontre entre auteur et spectateur et de celle entre le créateur et son public. Les différents objets ont presque tous leur singularité. C'est pourquoi l'établissement de règles qui permettent de comprendre l'expérience esthétique doit se faire avec prudence. Quelles sont les approches les plus pertinentes ? Y en a-t-il de vraiment pertinentes ? Faut-il en éliminer certaines ? Se questionner sur l'expérience esthétique demande aussi de s'interroger sur les catégories esthétiques dont nous disposons tous. Doit-on faire un choix?

Même si l'ouvrage est très synthétique et aborde une quantité d'œuvres non négligeable, Stéphane Olivesi a éliminé certaines approches. II n'oublie quasiment aucune période ou tout au moins s'intéresse aux mouvements essentiels, comme la Renaissance, le Baroque, les avant-gardes, le Romantisme, etc. II 